\title{
Equivalent Theories and Changing Hamiltonian Observables in General Relativity
}

\author{
J. Brian Pitts ${ }^{1}$ (D)
}

Received: 30 June 2017 / Accepted: 20 February 2018 / Published online: 17 March 2018

(C) The Author(s) 2018

\begin{abstract}
Change and local spatial variation are missing in Hamiltonian general relativity according to the most common definition of observables as having 0 Poisson bracket with all first-class constraints. But other definitions of observables have been proposed. In pursuit of Hamiltonian-Lagrangian equivalence, Pons, Salisbury and Sundermeyer use the Anderson-Bergmann-Castellani gauge generator $G$, a tuned sum of first-class constraints. Kuchař waived the 0 Poisson bracket condition for the Hamiltonian constraint to achieve changing observables. A systematic combination of the two reforms might use the gauge generator but permit non-zero Lie derivative Poisson brackets for the external gauge symmetry of General Relativity. Fortunately one can test definitions of observables by calculation using two formulations of a theory, one without gauge freedom and one with gauge freedom. The formulations, being empirically equivalent, must have equivalent observables. For de Broglie-Proca non-gauge massive electromagnetism, all constraints are second-class, so everything is observable. Demanding equivalent observables from gauge Stueckelberg-Utiyama electromagnetism, one finds that the usual definition fails while the Pons-SalisburySundermeyer definition with $G$ succeeds. This definition does not readily yield change in GR, however. Should GR's external gauge freedom of general relativity share with internal gauge symmetries the 0 Poisson bracket (invariance), or is covariance (a transformation rule) sufficient? A graviton mass breaks the gauge symmetry (general covariance), but it can be restored by parametrization with clock fields. By requiring equivalent observables, one can test whether observables should have 0 or the Lie derivative as the Poisson bracket with the gauge generator $G$. The latter definition is vindicated by calculation. While this conclusion has been reported previously, here the calculation is given in some detail.
\end{abstract}

\footnotetext{
$凶 \quad$ J. Brian Pitts jbp25@cam.ac.uk

1 Faculty of Philosophy, University of Cambridge, Cambridge, UK
} 
Keywords Hamiltonian formalism · Canonical quantization · Observables · Problem of time

\section{Problem of Missing Change and Spatial Variation in Observables}

Already in the mid-1950s there arose the problem of missing change in observables in the constrained Hamiltonian formulation of General Relativity: "There are indications that the Hamiltonian of the general theory of relativity may vanish and that all the observables are constants of the motion." Bergmann and Goldberg, [7] (see also [1]). This result appeared not too long after the introduction of novel distinctively Hamiltonian notions of gauge transformation and observables [9, Section 4], in contrast to the previously manifestly Lagrangian equivalent work [2]. Besides the "problem of time" due to missing change $[21,26]$, which owes much to how the Hamiltonian constraint $\mathcal{H}_{0}$ is treated, there is also a problem of space: local spatial variation is excluded by the condition for observables $\left\{O, \mathcal{H}_{i}\right\}=0$, pointing to global spatial integrals instead [51].

However, Bergmann was prepared to define observables in a variety of inequivalent ways; while his definition in terms of first-class secondary constraints is intrinsically Hamiltonian, at times he wanted a definition that was independent of the Hamiltonian formalism $[5,6,8]$. Relatedly, Pons, Salisbury and Sundermeyer have proposed a reformed definition of observables using the Anderson-Bergmann-Castellani gauge generator $G$, a tuned sum of all first-class constraints including the primaries $[2,11,39]$.

At times Bergmann and others have expected observables to be local or at least quasi-local [6, p. 250] [46, p. 115]. Kuchař, in the interests of finding real change, has been prepared to abolish altogether (not simply weaken) the requirement that observables have 0 Poisson bracket with what generates time gauge transformations (which he took to be the Hamiltonian constraint $\mathcal{H}_{0}$ ) [26,27]. According to Kiefer,

Functions $A(q, p)$ for which $\left\{A, \phi_{A}\right\} \approx 0$ holds are often called observables because they do not change under a redundancy transformation. It must be emphasized that there is no a priori relation of these observables to observables in an operational sense. This notion was introduced by Bergmann in the hope that these quantities might play the role of the standard observables in quantum theory (Bergmann 1961). [23, p. 105; see also p. 143]

By implementing the inevitable requirement that empirically equivalent theories have equivalent observables using the novel examples of massive photons and (formally) massive gravitons, this paper and its predecessor [35] reconsider the definition of observables and show that the conventional definition requires both the PonsSalisbury-Sundermeyer reform to use $G$ rather than separate first-class constraints and a novel non-zero Lie derivative Poisson bracket for external symmetries, partly inspired by Kuchař. As a result, observables are local 4-dimensional scalars, vectors, tensors, densities, etc., just as in Lagrangian/geometric formulations, including the metric and the curvature tensors. Thus change and local spatial variation are present after all.

The failure of observables to play their expected role has also led to circumvention by introducing new notions to do roughly the job that observables disappointingly 
didn't do $[16,42,49]$. It would be interesting to explore relations between these ideas and the reformed notion of observables.

\section{First-Class Constraints and Gauge?}

It is generally accepted that first-class constraints are related to gauge freedom, but there are two main views about what that precise relationship is. The original view, which retains manifest equivalence to the Lagrangian and which disappeared as the 1950s wore on and started reappearing around 1980, is that gauge transformations are generated by a tuned sum of first-class constraints (primary, secondary, etc.), the "gauge generator" $G[2,11,29,40,45,48]$. For Maxwell's electromagnetism the gauge generator is

$$
G(t)=\int d^{3} x\left(-\dot{\epsilon}(t, x) \pi^{0}+\epsilon(t, x) \pi^{i},{ }_{i}(t, x)\right) .
$$

Expressions are also known for General Relativity, both without and with the $3+1$ split $[2,11]$ This $G$-based view competes with what became the majority view (especially in books), that each first-class constraint FC alone generates a gauge transformation $[5,15,18,20,41]$.

While gauge transformations are not this paper's primary concern, gauge transformations and observables are naturally interrelated: observables ought to be invariant (or perhaps covariant) under gauge transformations, and transformations under which observables are invariant (or perhaps covariant) ought to be gauge transformations. Thus a revision of the notion of gauge transformation calls for a revision of the definition of observables [39], and to some degree vice versa.

Fortunately one can test definitions of observables by calculation using two formulations of a theory, one without gauge freedom and one with gauge freedom. The formulations, being empirically equivalent, must have equivalent observables. The equivalence of non-gauge and gauge formulations of massive quantum electrodynamics is presupposed in quantum field theory to show that the theory is renormalizable (shown using the Stueckelberg-Utiyama gauge formulation with a gauge compensation field) and unitary (shown using in effect the de Broglie-Proca formulation) [31, pp. 738, 739], [55, Chapter 21], [22, Chapter 10]. For de Broglie-Proca non-gauge massive electromagnetism, all constraints are second-class, so everything is observable. Demanding equivalent observables from gauge Stueckelberg-Utiyama electromagnetism, one can ascertain whether observables should have 0 Poisson bracket with each first-class constraint separately, or rather have 0 Poisson bracket only with the gauge generator $G$. It turns out that the usual definition fails while the Pons-SalisburySundermeyer definition with $G$ succeeds [35]. This result parallels arguments based on the requirement of Hamiltonian-Lagrangian equivalence [34].

\section{Internal Versus External Gauge Symmetries and Invariance Versus Covariance}

Definitions of observables often have been designed around electromagnetism, an internal symmetry, and imported into GR without much consideration for whether 
external symmetries might differ relevantly from internal ones [4,5,15]. In GR, $G$, acting on the 4-dimensional metric, gives the 4-d Lie derivative [11],

$$
£_{\xi} g_{\mu \nu}=\xi^{\alpha} g_{\mu \nu, \alpha}+g_{\mu \alpha} \xi^{\alpha},{ }_{\nu}+g_{\alpha \nu} \xi^{\alpha}, \mu
$$

The second and third terms are (for weak fields) analogous to the electromagnetic case, but the transport term $\xi^{\alpha} g_{\mu \nu},{ }_{\alpha}$ differentiates $g_{\mu \nu}$, thus making the transformation "external."

The use of $G$ does not suffice to yield changing and locally varying observables [35], at least not ones that one would expect on Lagrangian/geometric grounds such as the 4-metric. Because $G$ gives the 4-d Lie derivative, the definition of observables $\left\{O, G\left[\xi^{\alpha}\right]\right\}=0\left(\forall \xi^{\alpha}\right)$ implies $£_{\xi} O=0\left(\forall \xi^{\alpha}\right)$. Observables are not allowed to change in any direction, so $O$ is constant over time and space even with $G$. The problem of time is still present for observables even using $G$. At this point one might recall criticisms by Smolin and by Kuchař of the usual definition of observables, discussed above, as well as Bergmann's occasional view that observables should be local. Can one devise a systematic definition of observables that can also encounter a crucial test with the right examples?

One might wish to amend Kuchař's proposal in two ways (apart from using $G$ [39]). First, Kuchař's common-sense argument against $\left\{O, \mathcal{H}_{0}\right\}=0$ is just as compelling against $\left\{O, \mathcal{H}_{i}\right\}=0$ (which he retains), because spatial variation is as evident as change. Thus one should treat space and time alike and consider relaxing both $\left\{O, \mathcal{H}_{0}\right\}=0$ and $\left\{O, \mathcal{H}_{i}\right\}=0$. Second, abolishing any restrictions at all on time gauge behavior is unnecessarily strong, making any arbitrary behavior regarding time gauge transformations admissible. There is an overlooked intermediate position, not invariance but covariance, imposing some well-defined time coordinate transformation rule (scalar, vector, etc.). Infinitesimally, one would thus expect (especially after embracing $G$ ) to have a 4-dimensional Lie derivative, not 0 , be the result of the Poisson bracket in the definition of observables. One also notices that whereas electromagnetic gauge transformations are ineffable mental acts with no operational correlate (no knob or reading on a voltmeter), so electromagnetic observables must be invariant, general relativistic gauge (coordinate) transformations are already familiar from geography and daylight savings time. Being gauge-invariant does not require being the same at 1 a.m. Eastern Daylight Time and 1 a.m. Eastern Standard Time an hour later. Hence covariance rather than invariance is a reasonable criterion [33].

\section{Testing Definitions using Massive Gravity}

As massive electromagnetism comes in non-gauge and gauge versions, so does massive gravity. Non-gauge versions appeared in nonlinear form in the 1960s [17,30]. Gauge versions can be achieved by parametrization, promoting (or perhaps demoting [25]) preferred coordinates into fields varied in the action principle [3,36,43]. Massive gravity was rejected in the early 1970s due to either instability (spin 2-spin 0) or a discontinuous massless limit $[10,52,53,56]$. Progress was made on both fronts during the 2000s $[12,13,19]$, along with new challenges [14]. Fortunately, for present pur- 
poses it doesn't matter at all what problems massive gravity has. What matters is the relationship between the non-gauge and gauge versions. One might as well choose the version that makes the calculations the easiest, the Freund-Maheshwari-Schonberg (FMS) theory [17], which, when parametrized, gives nicest form, namely, minimal coupling of the scalar clock fields [43].

As with de Broglie-Proca massive electromagnetism, the observables in non-gauge massive gravity are obvious because all constraints are second-class [32]. Thus trivially everything has 0 Poisson bracket with all first-class constraints, making everything observable, including the 4-metric $g_{\mu \nu}$ and the non-zero momenta $\pi^{m n}$. The theory is merely Poincaré-invariant. The FMS mass term is

$$
\mathcal{L}_{m}=m^{2} \sqrt{-g}+m^{2} \sqrt{-\eta}-\frac{1}{2} m^{2} \sqrt{-g} g^{\mu v} \eta_{\mu \nu},
$$

where $\eta_{\mu \nu}=\operatorname{diag}(-1,1,1,1)$ in Cartesian coordinates. $\sqrt{-\eta}$ gives just a constant in the action.

One obtains the gauge version by parametrization, turning preferred Cartesian coordinates into clock fields $X^{A}(x)$, functions of arbitrary coordinates $x^{\mu}$. Only the mass term is affected. Now the reason for choosing the FMS massive theory becomes evident, namely, that its mass term, in contrast to the many other options out there (e.g., $[19,30])$, gives minimally coupled scalar clock fields in the expression

$$
\sqrt{-g} g^{\mu \nu} \eta_{A B} \frac{\partial X^{A}}{\partial x^{\mu}} \frac{\partial X^{B}}{\partial x^{\nu}}
$$

instead of using inverses, determinants, and/or fractional powers of $\eta_{A B} \frac{\partial X^{A}}{\partial x^{\mu}} \frac{\partial X^{B}}{\partial x^{v}}$, or sums thereof. It turns out that one can do calculations in the general case anyway [24], but that is a pleasant surprise. The parametrized mass term is

$$
\mathcal{L}_{m g}=m^{2} \sqrt{-g}+m^{2} \sqrt{-\eta}-\frac{1}{2} m^{2} \sqrt{-g} g^{\mu \nu} \eta_{A B} \frac{\partial X^{A}}{\partial x^{\mu}} \frac{\partial X^{B}}{\partial x^{\nu}},
$$

$\eta_{A B}=\operatorname{diag}(-1,1,1,1)$. (It isn't necessary to parametrize $\sqrt{-\eta}$ because the result is a total divergence.) This is just a cosmological constant $\sqrt{-g}$, a harmless constant $\sqrt{-\eta}$, and four minimally coupled scalar fields (one with the wrong sign). Gauge (parametrized) massive gravity becomes non-gauge massive gravity upon gauge-fixing $X^{A}-x^{\alpha}=0$.

Knowing that the 4-metric (and hence the inverse metric $g^{\mu v}$ ) is observable in the non-gauge theory, one can demand that observables in gauge massive gravity be equivalent to the non-gauge massive gravity observables. The equivalent quantity is $g^{\mu v} X^{A},{ }_{\mu} X^{B}{ }_{, v}$ : the gradients of the clock fields act as the tensor transformation law to the preferred Cartesian coordinates in which the non-gauge formulation is already expressed. By seeing how the quantity $g^{\mu \nu} X^{A},{ }_{\mu} X^{B},{ }_{\nu}$ behaves in parametrized massive gravity, we can learn how observables behave under external gauge transformations. Should observables satisfy $\{O, G[\xi]\}=0$, or $\{O, G[\xi]\} \sim £_{\xi} O$ (possibly just on-shell)? 


\subsection{Hamiltonian for Gauge Massive Gravity}

For General Relativity with minimally coupled scalar fields and a cosmological constant, the Poisson bracket 'algebra' of constraints is just as in GR [47]. For the parametrized version of the Freund-Maheshwarei-Schonberg theory, the same result therefore holds. It is straightforward to take the parametrized Lagrangian density and perform the constrained Legendre transformation with 4 minimally coupled scalars and $\Lambda$ GR $[28,47,54]$. In the ADM $3+1$ split, the 4-metric is broken into the lapse $N$, the shift vector $\beta^{i}$, and spatial metric $h_{i j}$. There are new canonical momenta for the clock fields:

$$
\pi_{A}=\frac{\partial \mathcal{L}_{m g}}{\partial X^{A}, 0}=-m^{2} \mathfrak{g}^{\mu 0} \eta_{A B} X^{B}, \mu
$$

Inverting, one gets

$$
\dot{X}^{A}=N \pi_{B} \eta^{A B} m^{-2} / \sqrt{h}+\beta^{i} X^{A},{ }_{i} .
$$

The Hamiltonian density is

$$
\begin{aligned}
\mathcal{H}_{m g}= & N\left(\mathcal{H}_{0}-m^{2} \sqrt{h}+\frac{\pi_{A} \pi_{B} \eta^{A B}}{2 m^{2} \sqrt{h}}+\frac{m^{2}}{2} \sqrt{h} h^{i j} X^{A},_{i} X^{B}{ }_{j}{ }_{j} \eta_{A B}\right) \\
& +\beta^{i}\left(\mathcal{H}_{i}+X^{A}{ }_{i} \pi_{A}\right)-m^{2} \sqrt{-\eta},
\end{aligned}
$$

with $\mathcal{H}_{0}$ and $\mathcal{H}_{i}$ as in GR. This expression has the same form as in GR (apart from an irrelevant constant $\sqrt{-\eta}$ ) if one defines a total (gravitational plus matter) Hamiltonian constraint

$$
\mathcal{H}_{0 T}=\mathcal{H}_{0}-m^{2} \sqrt{h}+\frac{\pi_{A} \pi_{B} \eta^{A B}}{2 m^{2} \sqrt{h}}+\frac{m^{2}}{2} \sqrt{h} h^{i j} X^{A}{ }_{, i} X^{B}{ }_{, j} \eta_{A B}
$$

and a total momentum constraint

$$
\mathcal{H}_{i T}=\mathcal{H}_{i}+X^{A},{ }_{i} \pi_{A}
$$

The Hamiltonian for parametrized massive gravity is (apart from terms involving primary constraints)

$$
\mathcal{H}_{m g}=N \mathcal{H}_{0 T}+\beta^{i} \mathcal{H}_{i T}-m^{2} \sqrt{-\eta}
$$

\subsection{Applying the Gauge Generator in Massive Gravity}

Avoiding velocities requires $3+1$ split of coordinate transformation descriptor $\xi^{\mu}$ [11,38]: $\epsilon=N \xi^{0}$ is primitive and so has 0 Poisson brackets; the same holds for $\epsilon^{i}=\xi^{i}+\beta^{i} \xi^{0}$. The primary constraints are as in General Relativity: $p$ conjugate to 
$N$ and $p_{i}$ conjugate to $\beta^{i}$ both vanish. The generator of changes of time coordinate in vacuum General Relativity is

$$
G[\epsilon, \dot{\epsilon}]=\int d^{3} x\left[\epsilon \mathcal{H}_{0}+\epsilon p_{j} h^{i j} N,{ }_{i}+\epsilon\left(N p_{i} h^{i j}\right),{ }_{j}+\epsilon\left(p N^{j}\right),{ }_{j}+\dot{\epsilon} p\right]
$$

This entity generates on phase space $\times$ time a transformation that, for solutions of the Hamiltonian field equations, changes the time coordinate in accord with 4-dimensional tensors. Given how the gauge generator can be built algorithmically starting with the primary constraints [37], one would expect the same expression for the gauge generator for parametrized massive FMS gravity, only with matter included in the secondary constraints. The Hamiltonian takes the form of GR $+\Lambda+$ minimally coupled scalars with altered matter-containing constraints $\mathcal{H}_{0 T}$ and $\mathcal{H}_{i T}$.

One can verify that the resulting modified expression for $G_{T}$ indeed generates gauge transformations; indeed displaying that calculation in more detail than appeared previously [35] is the aim of this paper. For the space-time metric there is no difference because matter does not couple to gravitational momenta. For the new matter fields one has

$$
\left\{G[\epsilon, \dot{\epsilon}], X^{A}(y)\right\}=-\epsilon(y) \pi_{B} \eta^{B A} /\left(m^{2} \sqrt{h}\right)=-\xi^{0} N \pi_{B} \eta^{B A} /\left(m^{2} \sqrt{h}\right) .
$$

Using the relation $\dot{X}^{A}=N \pi_{C} \eta^{A C} m^{-2} / \sqrt{h}+\beta^{i} X^{A},{ }_{i}$ recovered from $\dot{X}^{A}=\frac{\delta H}{\delta \pi_{A}}$, one gets

$$
\left\{G[\epsilon, \dot{\epsilon}], X^{A}(y)\right\}=-\xi^{0} X^{A},{ }_{0}+\xi^{0} \beta^{i} X^{A},{ }_{i}
$$

on-shell. This relates nicely to the Lie derivative of the scalar clock fields. The second term is involved in a cancellation.

The spatial gauge generator for vacuum GR is [11]

$$
G\left[\epsilon^{i}, \dot{\epsilon}^{i}\right]=\int d^{3} x\left[\epsilon^{i} \mathcal{H}_{i}+\epsilon^{i} N^{j}{ }_{i} p_{j}-\epsilon^{j}{ }_{i} N^{i} p_{j}+\epsilon^{i} N,{ }_{i} p+\epsilon^{i}, 0 p_{i}\right] .
$$

It generates 3-d spatial Lie derivatives of the 4-metric $g_{\mu \nu}$ even off-shell. Making the obvious alteration of the secondary constraint to include matter through $\mathcal{H}_{i T}$ gives the correct gauge generator, giving a Lie derivative of the scalar clock fields:

$$
\left\{G_{T}\left[\epsilon^{i}, \dot{\epsilon}^{i}\right], X^{A}(y)\right\}=\left\{\int d^{3} x \epsilon^{i}(x) X^{C},{ }_{i} \pi_{C}, X^{A}(y)\right\} .
$$

Going on-shell one gets the result

$$
-\left(\xi^{i}+\beta^{i} \xi^{0}\right) X^{A},{ }_{i}
$$

The off-shell result will be worked out below. 
The full gauge generator $G_{T}$ is the sum of these two parts [11]:

$$
G_{T}[\epsilon, \dot{\epsilon}]+G_{T}\left[\epsilon^{i}, \dot{\epsilon}^{i}\right]
$$

The vacuum gauge generator combination generates 4-dimensional coordinate transformations on the space-time metric, at least for solutions:

$$
\left\{G[\epsilon, \dot{\epsilon}]+G\left[\epsilon^{i}, \dot{\epsilon}^{i}\right], g^{\mu \nu}\right\}=-£_{\xi} g^{\mu \nu}
$$

Castellani [11], Pons et al. [38] (on-shell) in General Relativity. The new material parts of the total momentum constraint and total Hamiltonian constraint have no gravitational momenta and hence do not affect the space-time metric.

Acting on the clock fields $X^{A}$, the total generator gives (going on-shell eventually)

$$
\begin{aligned}
& \left\{G_{T}[\epsilon, \dot{\epsilon}]+G_{T}\left[\epsilon^{i}, \dot{\epsilon}^{i}\right], X^{A}(y)\right\}=-\xi^{0} X^{A},{ }_{0}+\xi^{0} \beta^{i} X^{A},{ }_{i}-\left(\xi^{i}+\beta^{i} \xi^{0}\right) X^{A},_{i} \\
& =-\xi^{0} X^{A},{ }_{0}-\xi^{i} X^{A},{ }_{i}=-\xi^{\mu} X^{A}, \mu
\end{aligned}
$$

the proper 4-dimensional expression for (minus) the Lie derivative of a scalar field with respect to the space-time vector field $\xi^{\mu}$ describing the infinitesimal coordinate transformation. One can thus see in outline how the whole of $g^{\mu \nu} X^{A},{ }_{\mu} X^{B},{ }_{\nu}$ behaves nicely, at least on-shell.

\subsection{Off-Shell Calculation in Detail}

It now being clear in general outline what to expect, one can profitably do the calculation with more detail and while remaining off-shell. The matter-inclusive spatial gauge generator is

$$
G_{T}\left[\epsilon^{i}, \dot{\epsilon}^{i}\right]=\int d^{3} x\left[\epsilon^{i} \mathcal{H}_{i T}+\epsilon^{i} N^{j},{ }_{i} p_{j}-\epsilon^{j},{ }_{i} N^{i} p_{j}+\epsilon^{i} N,{ }_{i} p+\epsilon^{i}, 0 p_{i}\right]
$$

One therefore has

$$
\begin{aligned}
& \left\{G_{T}\left[\epsilon^{i}, \dot{\epsilon}^{i}\right], g^{\mu \nu} X^{A},{ }_{\mu} X^{B}, \nu\right\}=\left\{G_{T}\left[\epsilon^{i}, \dot{\epsilon}^{i}\right], g^{\mu \nu}\right\} X^{A},{ }_{\mu} X^{B},{ }_{\nu} \\
& +g^{\mu \nu}\left\{G_{T}\left[\epsilon^{i}, \dot{\epsilon}^{i}\right], X^{A}, \mu\right\} X^{B}, \nu+B \leftrightarrow A
\end{aligned}
$$

using the Leibniz product rule,

$$
\begin{aligned}
= & \left\{G_{T}\left[\epsilon^{i}, \dot{\epsilon}^{i}\right], g^{\mu \nu}\right\} X^{A},{ }_{\mu} X^{B},{ }_{\nu}+\left(g^{m v}\left\{G_{T}\left[\epsilon^{i}, \dot{\epsilon}^{i}\right], X^{A}, m\right\}\right. \\
& \left.+g^{0 v}\left\{G_{T}\left[\epsilon^{i}, \dot{\epsilon}^{i}\right], X^{A}, 0\right\}\right) X^{B},{ }_{\nu}+B \leftrightarrow A
\end{aligned}
$$


splitting up space-time into space and time,

$$
\begin{aligned}
= & \left\{G_{T}\left[\epsilon^{i}, \dot{\epsilon}^{i}\right], g^{\mu \nu}\right\} X^{A},{ }_{\mu} X^{B},{ }_{\nu}+\left(g^{m \nu} \frac{\partial}{\partial x^{m}}\left\{G_{T}\left[\epsilon^{i}, \dot{\epsilon}^{i}\right], X^{A}\right\}\right. \\
& \left.+g^{0 \nu} \frac{\partial}{\partial x^{0}}\left\{G_{T}\left[\epsilon^{i}, \dot{\epsilon}^{i}\right], X^{A}\right\}\right) X^{B},{ }_{\nu}+B \leftrightarrow A
\end{aligned}
$$

pulling out the spatial derivative [50, p. 58] and using the Anderson-Bergmann velocity Poisson bracket for the 0th component [2],

$$
\begin{aligned}
= & \left\{G_{T}\left[\epsilon^{i}, \dot{\epsilon}^{i}\right], g^{\mu \nu}\right\} X^{A},{ }_{\mu} X^{B},{ }_{\nu}+\left[g^{m v} \frac{\partial}{\partial x^{m}}\left(-\epsilon^{i} X^{A},{ }_{i}\right)\right. \\
& \left.+g^{0 v} \frac{\partial}{\partial x^{0}}\left(-\epsilon^{i} X^{A},{ }_{i}\right)\right] X^{B},{ }_{v}+B \leftrightarrow A
\end{aligned}
$$

using the explicit form of the matter-enriched spatial gauge generator,

$$
\begin{aligned}
= & \left\{G_{T}\left[\epsilon^{i}, \dot{\epsilon}^{i}\right], g^{\mu \nu}\right\} X^{A},{ }_{\mu} X^{B}, \nu+\left[-g^{m v} £_{\epsilon} X^{A}, m\right. \\
& \left.+g^{0 v} \frac{\partial}{\partial x^{0}}\left(-£_{\epsilon} X^{A}\right)\right] X^{B},{ }_{\nu}+B \leftrightarrow A
\end{aligned}
$$

using the commutation of Lie and partial derivatives [44, p. 105] as applied to space rather than space-time.

The generator of time coordinate transformations is

$$
G_{T}[\epsilon, \dot{\epsilon}]=\int d^{3} x\left[\epsilon \mathcal{H}_{0 T}+\epsilon p_{j} h^{i j} N,{ }_{i}+\epsilon\left(N p_{i} h^{i j}\right),{ }_{j}+\epsilon\left(p N^{j}\right),{ }_{j}+\dot{\epsilon} p\right] .
$$

The temporal gauge generator thus acts on $g^{\mu \nu} X^{A},{ }_{\mu} X^{B},{ }_{\nu}$ as

$$
\begin{aligned}
& \left\{G_{T}[\epsilon, \dot{\epsilon}], g^{\mu v} X^{A},{ }_{\mu} X^{B}, v\right\}=\left\{G_{T}[\epsilon, \dot{\epsilon}], g^{\mu \nu}\right\} X^{A},{ }_{\mu} X^{B}, v \\
& +\left\{G_{T}[\epsilon, \dot{\epsilon}], X^{A},{ }_{\mu}\right\} X^{B},{ }_{\nu} g^{\mu \nu}+B \leftrightarrow A
\end{aligned}
$$

by the Leibniz product rule,

$$
=\left\{G_{T}[\epsilon, \dot{\epsilon}], g^{\mu v}\right\} X^{A},{ }_{\mu} X^{B},{ }_{\nu}+\left\{G_{T}[\epsilon, \dot{\epsilon}], X^{A}\right\}, \mu X^{B},{ }_{\nu} g^{\mu \nu}+B \leftrightarrow A
$$

by pulling out the spatial derivative and using the Anderson-Bergmann velocity Poisson bracket. Using the result for the clock fields $\left\{G_{T}[\epsilon, \dot{\epsilon}], X^{A}(y)\right\}=$ $-\epsilon(y) \pi_{B} \eta^{B A} /\left(m^{2} \sqrt{h}\right)$, one infers

$$
\begin{aligned}
& \left\{G_{T}[\epsilon, \dot{\epsilon}], g^{\mu \nu} X^{A},{ }_{\mu} X^{B}, v\right\}=\left\{G_{T}[\epsilon, \dot{\epsilon}], g^{\mu \nu}\right\} X^{A},{ }_{\mu} X^{B},{ }_{\nu} \\
& +\left(-\epsilon \pi^{A} /\left(m^{2} \sqrt{h}\right)\right), \mu X^{B},{ }_{\nu} g^{\mu \nu}+B \leftrightarrow A .
\end{aligned}
$$


The combined spatio-temporal gauge generator thus yields

$$
\begin{aligned}
& =\left\{G_{T}\left[\epsilon^{i}, \dot{\epsilon}^{i}\right]+G_{T}[\epsilon, \dot{\epsilon}], g^{\mu v} X^{A}, \mu X^{B}, v\right\}=-\left(£_{\xi} g^{\mu v}\right) X^{A}, \mu X^{B}, v \\
& -g^{\mu v} X^{B}, v \frac{\partial}{\partial x^{\mu}} \mathfrak{f}_{\epsilon} X^{A}-B \leftrightarrow A-\left(\epsilon \pi^{A} m^{-2} \sqrt{h}\right), \mu X^{B},{ }_{v} g^{\mu \nu}-B \leftrightarrow A \\
& =-\left(\mathfrak{f}_{\xi} g^{\mu v}\right) X^{A},{ }_{\mu} X^{B},{ }_{v}-g^{\mu v} X^{B}, v \frac{\partial}{\partial x^{\mu}}\left(\xi^{i} X^{A},{ }_{i}+\beta^{i} \xi^{0} X^{A},{ }_{i}\right. \\
& \left.+N \xi^{0} \pi^{A} m^{-2} \sqrt{h}\right)-B \leftrightarrow A \\
& =-\left(£_{\xi} g^{\mu v}\right) X^{A}, \mu X^{B},{ }_{v}-g^{\mu v} X^{B}, v \frac{\partial}{\partial x^{\mu}}\left(\xi^{i} X^{A},{ }_{i}+\xi^{0} \frac{\delta H}{\delta \pi_{A}}\right)-B \leftrightarrow A \\
& =-\left(\mathfrak{f}_{\xi} g^{\mu \nu}\right) X^{A}, \mu X^{B},{ }_{\nu}-g^{\mu \nu} X^{B},{ }_{\nu} \frac{\partial}{\partial x^{\mu}}\left(\xi^{v} X^{A},{ }_{v}-\xi^{0} X^{A},{ }_{0}+\xi^{0} \frac{\delta H}{\delta \pi_{A}}\right)-B \leftrightarrow A \\
& =-\left(£_{\xi} g^{\mu v} X^{A}, \mu X^{B}, v\right)-g^{\mu v} X^{B}, v \frac{\partial}{\partial x^{\mu}}\left(-\xi^{0} X^{A}, 0+\xi^{0} \frac{\delta H}{\delta \pi_{A}}\right)-B \leftrightarrow A
\end{aligned}
$$

The term that is not a 4-dimensional Lie derivative vanishes on-shell.

By the equivalence of the non-gauge and gauge observables, $g^{\mu \nu} X^{A},{ }_{\mu} X^{B},{ }_{\nu}$ must be observable in the gauge theory because $g^{\mu \nu}$ is observable in the non-gauge theory. Knowing that $g^{\mu \nu} X^{A},{ }_{\mu} X^{B},{ }_{\nu}$ must be an observable and calculating how $g^{\mu \nu} X^{A},{ }_{\mu} X^{B},{ }_{\nu}$ is acted upon by $G$, we learn that observables give a Lie derivative rather than 0 when one takes their Poisson bracket with $G$. Thus for observables one has

$$
\{G, O\}=-£_{\xi} O \neq 0
$$

on-shell, when $G$ generates coordinate transformations, an external symmetry.

Covariance, not invariance, suffices for the external gauge symmetry in this case. Thus the usual vanishing 0 Poisson bracket condition is wrong at least in this case. This case looks just like GR plus $\Lambda$ plus minimally coupled scalars, so the same result should hold there. Adding $\Lambda$ and minimally coupled scalars to GR is insignificant, so the same result should hold for GR itself. Thus quantities that change by a Lie derivative under Poisson bracket with the gauge generator are observable: scalar fields, vector fields, tensors, densities, etc.

Acknowledgements Funding was provided by John Templeton Foundation (Grant Nos. 38761, 60745).

Open Access This article is distributed under the terms of the Creative Commons Attribution 4.0 International License (http://creativecommons.org/licenses/by/4.0/), which permits unrestricted use, distribution, and reproduction in any medium, provided you give appropriate credit to the original author(s) and the source, provide a link to the Creative Commons license, and indicate if changes were made.

\section{References}

1. Anderson, J.L.: Absolute change in general relativity. In: Recent Developments in General Relativity, pp. 121-126. Pergamon and PWN, Oxford and Warsaw (1962)

2. Anderson, J.L., Bergmann, P.G.: Constraints in covariant field theories. Phys. Rev. 83, 1018-1025 (1951) 
3. Arkani-Hamed, N., Georgi, H., Schwartz, M.D.: Effective field theory for massive gravitons and gravity in theory space. Ann. Phys. 305, 96-118 (2003). arxiv:hep-th/0210184v2

4. Bergmann, P.G.: Introduction of "true observables" into the quantum field equations. Il Nuovo Cimento 3, 1177-1185 (1956)

5. Bergmann, P.G.: Observables in general relativity. Rev. Modern Phys. 33, 510-514 (1961)

6. Bergmann, P.G.: The general theory of relativity. In: Flügge, S. (ed.) Prinzipien der Elektrodynamik und Relativitätstheorie, Volume IV of Handbuch der Physik, pp. 203-272. Berlin, Springer (1962)

7. Bergmann, P.G., Goldberg, I.: Dirac bracket transformations in phase space. Phys. Rev. 98, 531-538 (1955)

8. Bergmann, P.G., Komar, A.: Observables and commutation relations. Les Théories Relativistes de la Gravitation, Royaumont, 21-27 Juin 1959, pp. 309-325. Centre National de la Recherche Scientifique, Paris (1962)

9. Bergmann, P.G., Schiller, R.: Classical and quantum field theories in the Lagrangian formalism. Phys. Rev. 89, 4-16 (1953)

10. Boulware, D.G., Deser, S.: Can gravitation have a finite range? Phys. Rev. D 6, 3368-3382 (1972)

11. Castellani, L.: Symmetries in constrained Hamiltonian systems. Ann. Phys. 143, 357-371 (1982)

12. de Rham, C., Gabadadze, G., Tolley, A.J.: Resummation of massive gravity. Phys. Rev. Lett. 106, 231101 (2011). arXiv:1011.1232v2 [hep-th]

13. Deffayet, C., Dvali, G., Gabadadze, G., Vainshtein, A.I.: Nonperturbative continuity in graviton mass versus perturbative discontinuity. Phys. Rev. D 65, 044026 (2002). arXiv:hep-th/0106001v2

14. Deser, S., Waldron, A.: Acausality of massive gravity. Phys. Rev. Lett., 110:111101 (2013). arXiv: 1212.5835

15. Dirac, P.A.M.: Lectures on Quantum Mechanics. Belfer Graduate School of Science, Yeshiva University. Dover reprint, Mineola, New York, 2001 (1964)

16. Dittrich, B.: Partial and complete observables for Hamiltonian constrained systems. General Relat. Gravit. 39, 1891-1927 (2007). arXiv:gr-qc/0411013

17. Freund, P.G.O., Maheshwari, A., Schonberg, E.: Finite-range gravitation. Astrophys. J. 157, 857-867 (1969)

18. Govaerts, J.: Hamiltonian Quantisation and Constrained Dynamics. Leuven Notes in Mathematical and Theoretical Physics 4B. Leuven University Press, Leuven (1991)

19. Hassan, S.F., Rosen, R.A.: On non-linear actions for massive gravity. J. High Energy Phys. 1107(009) (2011) arXiv:1103.6055v3 [hep-th]

20. Henneaux, M., Teitelboim, C.: Quantization of Gauge Systems. Princeton University Press, Princeton (1992)

21. Isham, C.J.: Canonical quantum gravity and the problem of time. In: Ibort, L.A., Rodríguez, M.A. (eds.) Integrable Systems, Quantum Groups, and Quantum Field Theories, pp. 157-287. Kluwer, Dordrecht. Lectures presented at the NATO Advanced Study Institute "Recent Problems in Mathematical Physics," Salamanca, June 15-27, 1992 (1993) arxiv:gr-qc/9210011

22. Kaku, M.: Quantum Field Theory: A Modern Introduction. Oxford University, New York (1993)

23. Kiefer, C.: Quantum Gravity, 3rd edn. Oxford University Press, Oxford (2012)

24. Klusoň, J.: Hamiltonian analysis of the Higgs mechanism for graviton. Class. Quantum Gravity 28 , 155014 (2011). arXiv:1005.5458 [hep-th]

25. Kuchař, K.: Canonical quantization of gravity. In: Israel, W. (ed.) Relativity, Astrophysics, and Cosmology, pp. 237-288. D. Reidel, Dordrecht (1973)

26. Kuchař, K.V.: Time and interpretations of quantum gravity. In: Kunstatter, G., Vincent, D., Williams, J. (eds.) Proceedings of the 4th Canadian Conference on General Relativity and Relativistic Astrophysics, pp. 211-314. World Scientific, Singapore (1992)

27. Kuchař, K.V.: Canonical quantum gravity. In: Gleiser, R.J., Kozameh, C.N., Moreschi, O.M. (eds.) General Relativity and Gravitation 1992: Proceedings of the Thirteenth International Conference on General Relativity and Gravitation held at Cordoba, Argentina, 28 June-4 July 1992, pp. 119-150. Institute of Physics Publishing, Bristol (1993) arXiv:gr-qc/9304012

28. Misner, C., Thorne, K., Wheeler, J.A.: Gravitation. Freeman, New York (1973)

29. Mukunda, N.: Generators of symmetry transformations for constrained Hamiltonian systems. Physica Scripta 21, 783-791 (1980)

30. Ogievetsky, V.I., Polubarinov, I.V.: Interacting field of spin 2 and the Einstein equations. Ann. Phys. 35, 167-208 (1965) 
31. Peskin, M.E., Schroeder, D.V.: An Introduction to Quantum Field Theory. Addison-Wesley, Reading (1995)

32. Pitts, J.B.: Constrained dynamics of universally coupled massive spin 2-spin 0 gravities. J. Phys. 33, 279-284 (2006) arXiv:hep-th/0601185, Talk given at QG05, Cala Gonone, Sardinia, Italy, September 2005

33. Pitts, J.B.: Change in Hamiltonian general relativity from the lack of a time-like Killing vector field. Stud. Hist. Philos. Modern Phys. 47, 68-89 (2014a). arXiv:1406.2665

34. Pitts, J.B.: A first class constraint generates not a gauge transformation, but a bad physical change: the case of electromagnetism. Ann. Phys. 351, 382-406 (2014b). arXiv:1310.2756

35. Pitts, J.B.: Equivalent theories redefine Hamiltonian observables to exhibit change in general relativity. Class. Quantum Gravity, 34(055008) (2017). https://doi.org/10.1088/1361-6382/aa5ce8. arXiv:1609.04812 [gr-qc]

36. Pitts, J.B., Schieve, W.C.: Universally coupled massive gravity. Theor. Math. Phys. 151, 700-717 (2007). arXiv:gr-qc/0503051v3

37. Pons, J.M.: On Dirac's incomplete analysis of gauge transformations. Stud. Hist. Philos. Modern Phys. 36, 491-518 (2005). arXiv:physics/0409076v2

38. Pons, J.M., Salisbury, D.C., Shepley, L.C.: Gauge transformations in Einstein-Yang-Mills theories. J. Math. Phys. 41, 5557-5571 (2000). arXiv:gr-qc/9912086

39. Pons, J.M., Salisbury, D.C., Sundermeyer, K.A.: Observables in classical canonical gravity: Folklore demystified. J. Phys. 222, 012018 (2010) First Mediterranean Conference on Classical and Quantum Gravity (MCCQG 2009). arXiv:1001.2726v2 [gr-qc]

40. Rosenfeld, L.: Zur Quantelung der Wellenfelder. Annalen der Physik, 397, 113-152. Translation by Donald Salisbury and Kurt Sundermeyer, "On the Quantization of Wave Fields," European Physical Journal H42 (2017), pp. 63-94. https://doi.org/10.1140/epjh/e2016-70041-3

41. Rothe, H.J., Rothe, K.D.: Classical and Quantum Dynamics of Constrained Hamiltonian Systems. World Scientific, Hackensack (2010)

42. Rovelli, C.: Partial observables. Phys. Rev. D 65, 124013 (2002). arXiv:gr-qc/0110035

43. Schmelzer, I.: General ether theory (2000). arXiv:gr-qc/0001101

44. Schouten, J.A.: Ricci-Calculus: An Introduction to Tensor Analysis and Its Geometrical Application. 2nd edn. Springer, Berlin (1954). https://doi.org/10.1007/978-3-662-12927-2

45. Shepley, L.C., Pons, J.M., Salisbury, D.C.: Gauge transformations in general relativity-A report. Turk. J. Phys., 24(3), 445-452 (2000). Regional Conference on Mathematical Physics IX, 9-14 Aug. 1999, Istanbul, Turkey

46. Smolin, L.: The present moment in quantum cosmology: Challenges to the arguments for the elimination of time. arxiv:gr-qc/0104097. Slightly revised version of essay published in Robin Durie, ed., Time and the Instant, Clinamen Press, Manchester (2000) pp. 112-143 (2001)

47. Sundermeyer, K.: Constrained Dynamics: With Applications to Yang-Mills Theory, General Relativity, Classical Spin, Dual String Model. Lecture Notes in Physics, vol. 169. Springer, Berlin (1982)

48. Sundermeyer, K.: Symmetries in Fundamental Physics, 2nd edn. Springer, Heidelberg (2014)

49. Tambornino, J.: Relational observables in gravity: a review. SIGMA 8(017) (2012). arXiv:1109.0740

50. Thiemann, T.: Lectures on loop quantum gravity. In: Giulini, D.J.W., Kiefer, C., Lämmerzahl, C. (eds.) Quantum Gravity: From Theory to Experimental Search, number 631 in Lecture Notes in Physics, pages 41-135. Springer, Berlin. gr-qc/0210094, based on lectures given at the 271st WE Heraeus Seminar "Aspects of Quantum Gravity: From Theory to Experimental Search", Bad Honnef, Germany, February 25th - March 1st, 2002 (2003)

51. Torre, C.G.: Gravitational observables and local symmetries. Phys. Rev. D 48, R2373-R2376 (1993)

52. van Dam, H., Veltman, M.: Massive and mass-less Yang-Mills and gravitational fields. Nuclear Phys. B 22, 397-411 (1970)

53. van Dam, H., Veltman, M.: On the mass of the graviton. General Relat. Gravit. 3, 215-220 (1972)

54. Wald, R.M.: General Relativity. University of Chicago, Chicago (1984)

55. Weinberg, S.: The Quantum Theory of Fields, Volume II: Modern Applications. Cambridge University Press, Cambridge (1996)

56. Zakharov, V.I.: Linearized gravitation theory and the graviton mass. J. Exp. Theor. Phys. Lett. 12, 312-314 (1970) 